\title{
Proteogenomic studies in epithelial ovarian cancer: established knowledge and future needs
}

There has been a concerted effort over the last decade to improve our understanding of the complex biology of ovarian cancer. A linear growth in published proteogenomic studies has addressed a variety of questions regarding its molecular pathogenesis. A number of genes have been identified by transcriptomic approaches, some of which are being investigated as putative tumor markers (HE4, OPN, $E p-C A M$ and Mesothelin), whilst others are potential targets for molecular therapeutic approaches (VEGF, 104, EGFR, MUC1, CLDN4 and SLPI). Proteogenomics has the potential to further change our current characterization and treatment of ovarian cancer. Additional advances will depend on integrated study designs, interdisciplinary collaborations, use of robust high-throughput platforms, as well as uniform guidelines for bioinformatic analyses.

\section{KEYWORDS: biomarkers diagnostics glycomics microarray ovarian cancer ovary post-translational modifications profiling proteomics transcriptomics}

Epithelial ovarian cancer is the fourth most common cause of cancer mortality in women in the western world and the leading cause of death from gynecological malignancies [1]. The majority of women have advanced-stage disease at initial diagnosis and a 5-year survival of $10-30 \%$ [2]. The median survival after recurrence is only 2 years despite advances in chemotherapy and secondary debulking surgery in selected patients $[1,3,4]$. We clearly need better insights into the biology of the disease in order to detect the disease at an earlier, potentially curable stage and to develop more targeted and effective treatment strategies.

Epithelial ovarian cancers are made up of five main histological subtypes that vary in their biological behavior, response to treatment and overall prognosis [5]. However, even within specific histological subtypes there is considerable heterogeneity, particularly within the more common serous cancers [6,7]. Although International Federation of Gynecology and Obstetrics (FIGO) stage and grade are still used to classify all ovarian cancers, it has been recently recognized that there are two separate and quite distinct subgroups of serous cancer: Type 1, which are low grade and indolent and have a distinct molecular pathogenesis, and the more common Type 2 serous cancers, which are high grade and disseminate rapidly [8,9]. Type 1 tumors appear to arise from the ovarian surface epithelium or inclusion cysts [10] and follow a stepwise progression through microinvasive tumors and tumors of low malignant potential.
They commonly have mutations in KRAS and $B R A F$ [9]. By contrast, Type 2 serous tumors appear to arise from the fimbrial end of the fallopian tube [11-14] and $p 53$ mutations occur early. BRCA1/2-associated ovarian cancers in particular appear to arise from the fimbrial end of the fallopian tube and have a better response to platinum-based chemotherapy [15-17].

Despite the genetic distinction between the various ovarian cancer histological subtypes [18], they are commonly treated in the same manner: namely, with maximal cytoreductive surgery [19-22] followed by platinum- and taxane-based chemotherapy $[4,23,24]$. It would be valuable if we could use molecular expression profiles to classify ovarian tumors into distinct subtypes based on the biological behavior and to better predict individual patient outcomes based on a such a personal molecular 'biosignature' $[25,26]$. Good examples of this approach have already been provided for breast cancers and lymphomas $[27,28]$.

There has been a concerted effort over the last decade to identify specific molecular profiles of ovarian cancers using cDNA microarrays and oligonucleotide arrays [29,30]. These highthroughput technologies for transcriptomics or genomic expression profiling permit the analysis of the expression of thousands of genes in one experiment examining a specific biological system [31]. The resulting patterns allow researchers to investigate important biological questions that have not been easily addressed with traditional gene expression technologies such as Northern
Francis Jacob ${ }^{1}$,

Darlene R Goldstein ${ }^{2,3}$,

Daniel Fink ${ }^{4} \&$ Viola

Heinzelmann-Schwarz ${ }^{1 \dagger}$

${ }^{\dagger}$ Author for correspondence:

${ }^{1}$ Translational Research Group,

Department of Gynecology,

University Hospital Zurich,

Nord I D222,

Frauenklinikstrasse 10,

8091 Zurich, Switzerland

Tel.: +41 442555 374;

Fax: +41 442554 553;

viola@heinzelmann.ch

${ }^{2}$ Institut de mathématiques,

Ecole Polytéchnique Fédérale,

Lausanne, Switzerland

${ }^{3}$ Swiss Institute of

Bioinformatics, Lausanne,

Switzerland

${ }^{4}$ Department of Gynecology,

University Hospital Zurich,

Switzerland 
blots, in situ hybridization or RNase protection assays. Following the development of large-scale profiling technologies, the first high-throughput studies examining protein expression levels were performed using serological analysis of autologous tumor antigens by recombinant cDNA expression cloning (SEREX) [32]. Within the growing spectrum of proteomic technologies, most techniques were based on 2D polyacrylamide gel electrophoresis (2D-PAGE) mainly in combination with mass spectrometry techniques such as matrix-assisted laser desorption/ ionization-time of flight (MALDI-TOF), surface-enhanced laser desorption/ionization-time of flight (SELDI-TOF), electrospray ionization (ESI) or with liquid chromatography (LC).

There has been a linear growth in the number of publications on 'biomic' studies of ovarian cancer. Since the initial studies on transcriptomics of ovarian cancer in 1999 [33-35], 237 studies were published until the end of 2007 using various -omic technologies. A vast amount of data has been amassed over the last decade, providing greater insight into the heterogeneous genetic background of ovarian cancer. This has led to the identification of novel markers as well as new diagnostic, prognostic and therapeutic targets.

The aim of this report is to provide a comprehensive summary of the totality of published studies pertaining to functional genomics of ovarian cancer. We attempt to put the stateof-the-art of proteogenomic studies in ovarian cancer into context, showing the co-evolution of ovarian cancer knowledge and technologies for new assays, which provide the basis for our view of the direction of future research. We also summarize the data and review the clinical questions addressed in the published studies and highlight the most promising novel tumor markers. We briefly describe new technical possibilities [36], which have the potential both to improve the early detection and better predict prognosis of patients with epithelial ovarian cancer, and take a glimpse at the future of ovarian cancer biomarker research.

\section{Identification of genomic profiling studies}

We performed an electronic search of the online databases PubMed [101], Cochrane [102] and Medline [103] to identify publications on ovarian cancer proteogenomic profiling during the 9-year period, 1999-2007. Keywords used to search the databases were, in changing combinations: ovarian cancer, ovary, human ovarian; in combination with: mass spectrometry (MS), serial analysis of gene expression (SAGE), SEREX, cDNA microarray, differential methylation hybridization (DMH), comparative genomic hybridization $(\mathrm{CGH})$, array $\mathrm{CGH}$ $(\mathrm{aCGH})$, expression profiles, oligonucleotide array, proteomics, transcriptomics, genomics; or: expression profiling, array, microarray and single-nucleotide polymorphism (SNP) array. Only published studies that used humanderived cell lines or human clinical samples were included. Articles were excluded when the term 'microarray' was related to tissue microarrays without further mentioning expression profiling within the paper; the data were from another, already published proteogenomic study; and publications in languages other than English.

The identified publications were divided into three categories: transcriptomics, proteomics, and other profiling technologies. Transcriptional profiling includes cDNA and oligonucleotide microarray studies and SAGE; proteomic profiling includes studies using SEREX, 2D PAGE, MALDI-TOF, SELDI-TOF, ESI and LC; and other profiling technologies cover DMH, SNP arrays and aCGH studies. The publication data are summarized according to year of publication, high-throughput technology used, number of profiled samples, sample type, sample preparation, aim of study and main study findings (we provide this large summary as supplementary data). 'Numbers of studies' as listed in the supplementary data do not reflect the numbers of published studies but the research questions addressed within studies, with multiple questions possibly arising from individual proteogenomic datasets.

Data summaries were further descriptively analyzed for the overall time of 9 years and for three individual time periods of 3 years each. We evaluated the whole time period in order to study the trend of profiling studies performed per year and method used (FIgure 1). Individual 3-year periods were used to demonstrate trends within sample types (Figure 2), high-throughput technologies (Figure 3), and research questions addressed (Figure 4). Individual research questions were also further subclassified into 13 main categories (Figure 4):

- Tumor marker discovery (cancer vs normal);

- Treatment effects (treatment; wild-type vs knock out or treated vs untreated), resistance (drug resistance); Patient outcome (outcome); 


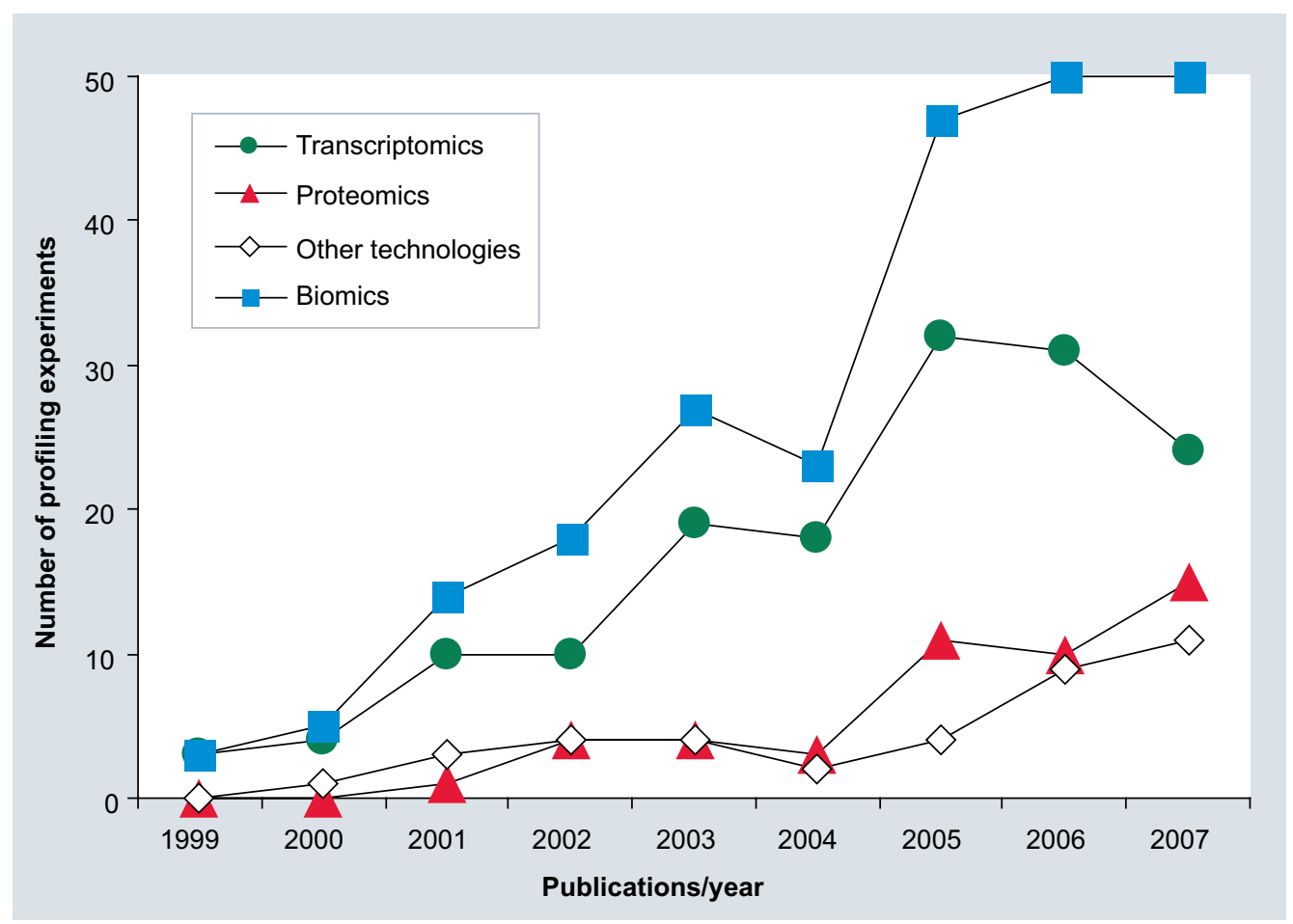

Figure 1. Development of biomics in ovarian cancer from 1999-2007. Absolute numbers of profiling experiments published over the last 9 years. All incorporated techniques are grouped within the 'Biomics' graph and individually for 'transcriptomics' (CDNA microarray, oligonucleotide array and SAGE), 'proteomics' (SEREX, 2D PAGE, protein microarray, SELDI -, ESI- and MALDI-TOF MS and their modified techniques) and 'other technologies' (SAGE, SNP array, CGH and DMH).

2D PAGE: 2D polyacrylamide gel electrophoresis; CDNA: Complementary DNA; CGH: Comparative genomic hybridization; DMH: Differential methylation hybridization; ESI: Electrospray ionization; MALDI-TOF: Matrix-assisted laser desorption/ionization-time of flight; MS: Mass spectrometry; SAGE: Serial analysis of gene expression; SELDI: Surface enhanced laser desorption/ionization; SEREX: Serological analysis of recombinant tumor CDNA expression libraries; SNP: Single-nucleotide polymorphism.

- Distinction of different histological subtypes (subtypes), histological grades (grades), the International Federation of Gynecology and Obstetrics (FIGO) stage (stages), primary versus metastatic site (metastasis); ovarian versus nonovarian cancers (epithelial ovarian cancer vs non-epithelial ovarian cancer);

- Aberrant chromosomal patterns (chromosomes), epigenetics (epigenetics), hereditary diseases (hereditary);

- Specificity of technique (technical optimization).

Ovarian cancers subtypes as defined by histology, stage, grade, organ of origin and outcome were further studied in order to assess whether a genetic distinction between groups would be justified. Taken together, Figures 1-4 trace the history of clinical biomarker identification using genomic and proteomic technologies. These figures also serve to emphasize the point that although the amount of data

is increasing, we still face the daunting challenge to translate the information contained therein to clinically relevant knowledge that will benefit patients.

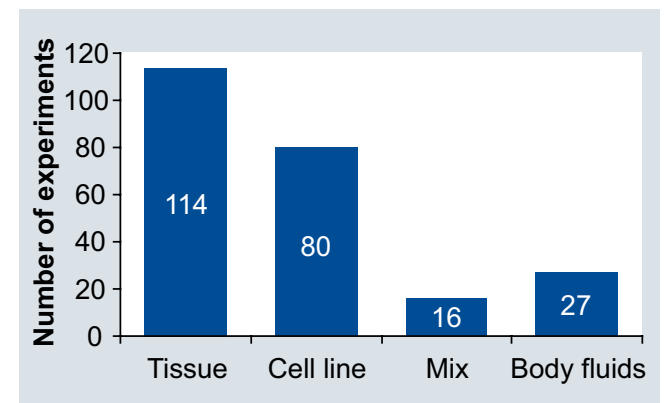

Figure 2. Variation of profiled biological samples using high-throughput technologies. Samples used for profiling experiments analyzed within this study: tissue samples ('tissue', $n=114$ ), ovarian cancer cell lines ('cell line', $n=80$ ), and a mix of both ('mix', $n=16)$. 'Body fluids' $(n=27)$ stands for biological samples from blood, pleural effusions or ascites. 


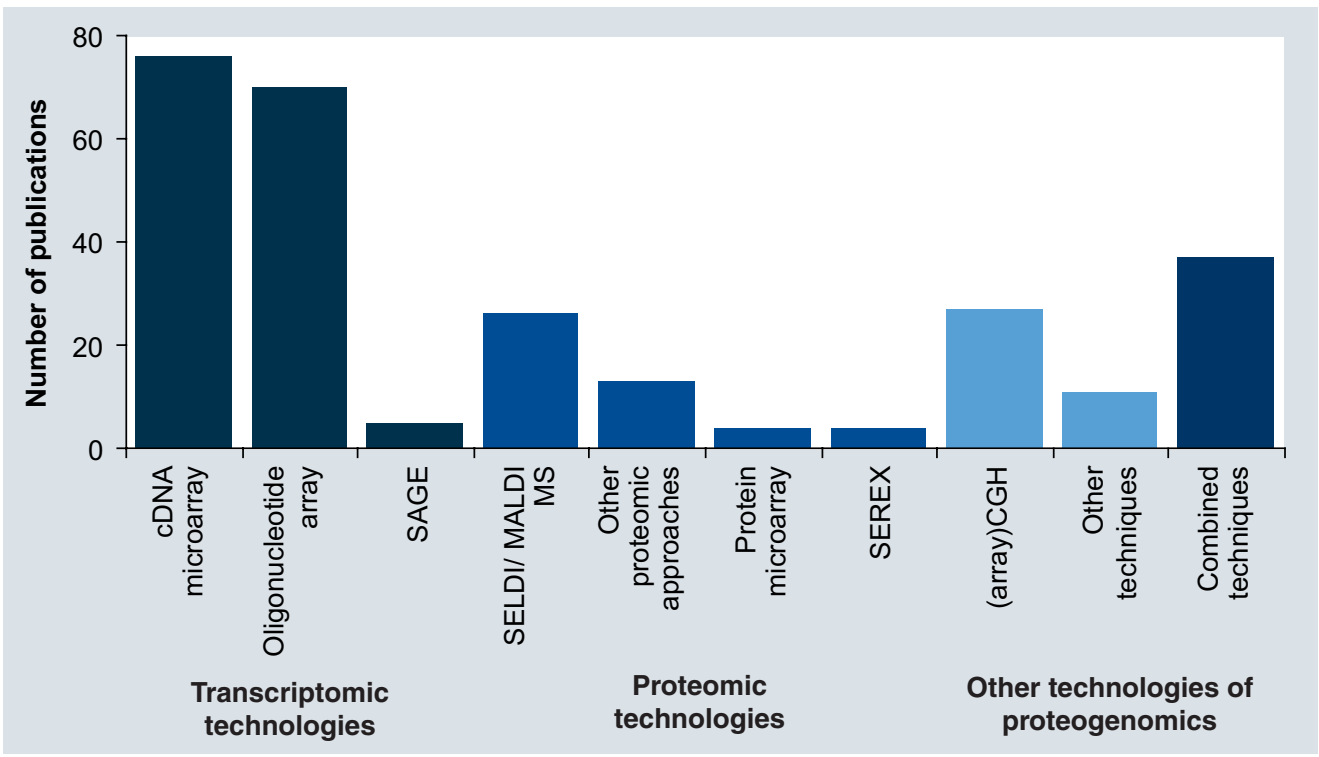

Figure 3. Distribution in ovarian cancer profiling studies by high-throughput technology applied. Numbers of publications using specific high-throughput technologies are shown. 'Combined techniques' represents the publications using more than one high-throughput technique.

We incorporated published lists of new candidate tumor markers derived from transcriptomic approaches for distinguishing between healthy individuals and patients with ovarian cancer into our previously described automated over-lapping ovarian (OLOV) database [37]. All published candidate gene lists were screened for gene overlaps across individual studies, with overlaps identified by matching gene symbols, accession numbers and UniGene identifiers. We used Gene Cards [104] and the Human Protein Reference database [105] further enhance the list of most frequently identified potential tumor marker genes, with the addition of information regarding cellular localization, translational modifications, molecular class, glycosylation as post-translational modification, available treatment options and clinical testing status (Phase I-III trials). The numbers of studies demonstrating a significant difference between research question groups are presented graphically using OLOV as described previously [37]. OLOV-incorporated genes were ranked by previously described gene identifiers and sorted in decreasing order by number of reoccurrence across studies.

\section{Development of high-throughput profiling studies}

Between the years 1999 and 2007, 237 primary studies addressing functional 'biomics' in ovarian cancer have been published. During the first 5 years, there is a roughly linear growth in the number of publications using profiling techniques, after which the number of publications reaches a plateau (Figure 1). Two thirds of studies between 1999 and 2004 used transcriptomic (rather than proteomic or other) methods. After a clear increase in 2005, a steady decline in number of transcriptomic studies can be observed, which is linked to a nearly linear increase in proteomic and other methods since 2004. These other methods were used as frequently as proteomics methods. Although Minimal Information About a Microarray Experiment (MIAME) guidelines state the need to openly distribute microarray raw data for further validation or meta-analysis [38,39], most frequently only lists of significant gene expressions are published, particularly for the earlier time periods. Unfortunately, such lists are of limited utility for integrating information across studies.

\section{Profiling technologies used in ovarian cancer}

We have grouped profiling technologies into three major classes:

- Transcriptomics (63.7\% of studies): for example, cDNA and oligonucleotide microarrays and SAGE technology, used to study gene expression;

- Proteomics (20.3\% of studies): 2D PAGE, typically in conjunction with mass spectrometry technologies, SEREX and protein microarray;

- Other technologies (16.0\% of studies), including: (array) CGH, SNP array and DMH. 
Multiple techniques were used in $15.6 \%$ of studies, most of which combine either transcriptomics with CGH (29.7\%), or proteomic technologies with transcriptomics (5\%) (Figure 3).

The majority $(63.7 \%)$ of ovarian cancer-specific questions were addressed using technologies to identify gene expression patterns, including cDNA microarrays (51.3\%) and oligonucleotide (46.3\%) arrays, most commonly produced by Affymetrix (Santa Clara, California, USA; HuGeFL, U95- and U133-series).

Robust transcriptomic technologies and analysis methodologies have led to the detection of several new ovarian cancer biomarkers. By contrast, even with advances in mass spectrometry, proteomic approaches have led to only a few protein biomarkers that are used clinically. Despite technical complexity, various recent reviews have discussed the great potential of proteomics in clinical biomarker discovery [40-44]. Proteomic approaches applied to detecting clinical biomarkers typically involve a broader spectrum of samples and larger chemical information content on the protein level than other types of biomic studies [45], but technical challenges have hindered development. Owing to the broad and complex diversity of proteins, the field of proteomics has to deal with a wide range of differences in molecular size, relative abundance, concentration, polarization and ionization as well as the high dynamic concentration range of proteins in complex samples like tissues and body fluids. Since there is not at present a single, optimal instrument that satisfies all proteomics research needs, there is a wide variety of mass spectrometry instrumentations, including an extensive assortment of modular arrangements of different types of mass analysers [46]. The current standards in bioinformatical analyses [47] and normalization procedures [48] are rather limited.

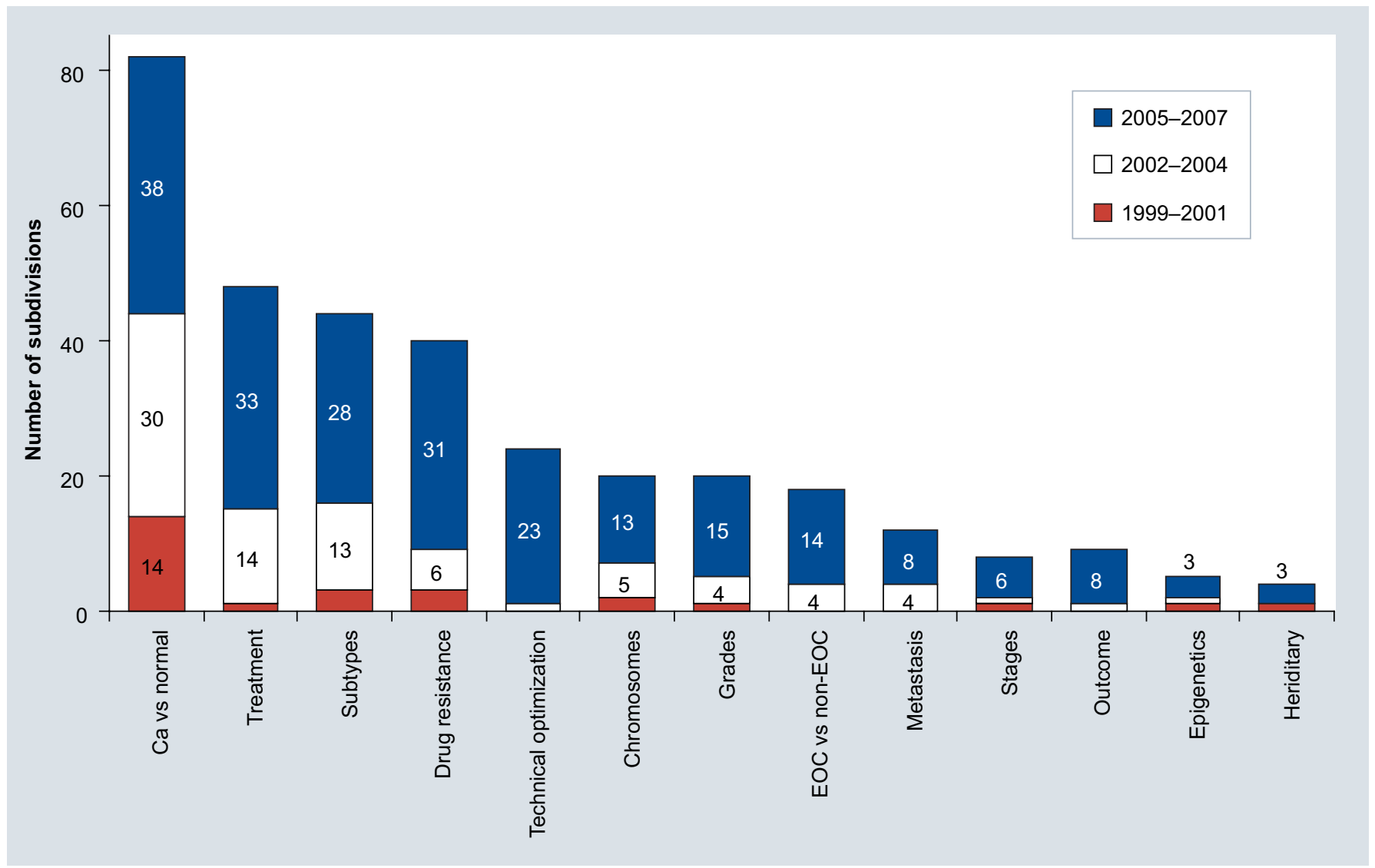

Figure 4. Categorization of investigated aims in ovarian cancer proteogenomic studies from 1999 to 2007. Research questions addressed within proteogenomics studies on ovarian cancer are grouped within 3-yearly time periods as (A) tumor marker discovery (Ca vs normal), (B) treatment effects (treatment; wild-type vs knock-out or treated vs untreated), resistance (drug resistance), patient outcome (outcome), (C) distinction of different histological subtypes (subtypes), grades (grades), stages (stages), primary versus metastatic site (metastasis), ovarian vs nonovarian cancers (EOC vs non-EOC), (D) aberrant chromosome patterns (chromosomes), epigenetics (epigenetics), hereditary diseases (hereditary), and (E) specificity of technique (technical optimization). Numbers within graphs represent numbers of studies within time period and question addressed.

Ca: Cancer; EOC: Epithelial ovarian cancer. 
Recent advances in mass spectrometry-based quantitative proteomics permit the identification of differentially expressed proteins in cells, tissues and body fluids in a high-throughput manner [49,50]. Quantification of proteins by mass spectrometry is currently most commonly performed by differential isotopic labeling of samples, separation of the peptides by liquid chromatography and identification as well as quantification of the peptides by shotgun mass spectrometry and database searches [51-54]. Major improvements during recent years have been achieved by:

- Label-free quantifications that integrate the total ion current of peptide signals as quantitative measure of original peptide concentration [55-58];

- Selected reaction monitoring for quantitative protein identification [59-61];

- Protein microarrays as protein-detecting microarrays, sandwich immunoassays with spotted antibodies, antigen capture immunoassays and direct immunoassays [62].

\section{Variation of biological sample types}

The most commonly profiled samples in transcriptomic studies consisted of human tissue (48.3\%), cell lines $(41.7 \%)$ or both tissues and cell lines $(9.3 \%)$, with a small minority $(0.7 \%)$ using body fluids. During recent years a change in sample selection has taken place; individual samples were required to be more uniform so that tumors with mixed histological appearances would tend to be excluded. This constraint facilitates laser capture microdissection (LMD) [63] for paraffin-embedded or fresh-frozen tissue specimens in cancer as well as for normal controls [64], particularly ovarian surface epithelium. However, the rate of studies incorporating LMD in order to allow homogenous messenger RNA generation was only $8.9 \%$. Proteomic experiments were performed on tissue specimens (14.6\%), cell lines $(31.2 \%)$, and body fluids (e.g., blood serum, ascites or pleural effusions; $54.2 \%$ ). The first studies examining ascites and ovarian cyst fluid have only recently been published [65].

The selection of normal control tissue is a major concern in most of the studies published. Whilst in earlier studies whole ovaries were used as a normal control or human ovarian surface epithelial cell lines were used in comparison to tissue samples, improvements have been under way using laser capture microdissection for human ovarian surface epithelium. Whilst quantities of ovarian surface epithelial cells are a major problem, new proposed precursor lesions such as inclusion cysts and, more recently, the tubal epithelium $[10-12,14,66]$ have so far only rarely been incorporated into biomic studies.

\section{Study aims within genomic profiling}

Study aims for all 237 publications were characterized and grouped, and are shown as absolute numbers in a bar graph (Figure 4). As study aims have changed during the years, they are presented in 3-yearly intervals (1999-2001, 2002-2004 and 2005-2007), thus demonstrating the general interest shift over time. During these three time periods, most studies investigated the differentiation between cancer versus normal in order to find new biomarkers (82 studies), experimental treatment settings (48 studies), histological subtypes (44 studies), tumor grades (20 studies) and chromosomes (20 studies) aberrant in ovarian cancer (Figure 4). The search for a new tumor marker in ovarian cancer (cancer vs normal) was the most studied aspect during 2005-2007 in 38 studies, nearly the same as during the previous 3-year period (2002-2004, 30 studies). The other most commonly addressed topics during the past 3 years included treatment effects (33 studies), drug resistance (31 studies), histological subtype distinction (28 studies), technical optimization (23 studies), grades (15 studies) and chromosomes (13 studies). There is a growing diversity during this most recent period, with the emergence of new fields such as technical optimization of experimental procedures.

\section{Distinct ovarian cancer biology identified by high-throughput profiling}

Clinico-pathological characteristics of ovarian cancer were examined on the molecular level to define distinct proteogenomic patterns. Studies aimed to identify a pattern for histological subtypes, stage, grade, organ of origin and disease progression. Distinctions identified for particular subgroups of ovarian tumors are shown in Figure 5. These results show that there is a clear molecular difference between histological subtypes of mucinous, clear cell and endometrioid ovarian cancers. Furthermore, endometrioid cancers of either ovarian or endometrial origin seem to have a distinct molecular pattern, even when expressing the same histological phenotype. Serous ovarian cancers of different grades, especially serous borderline tumors, are biologically different from serous ovarian 


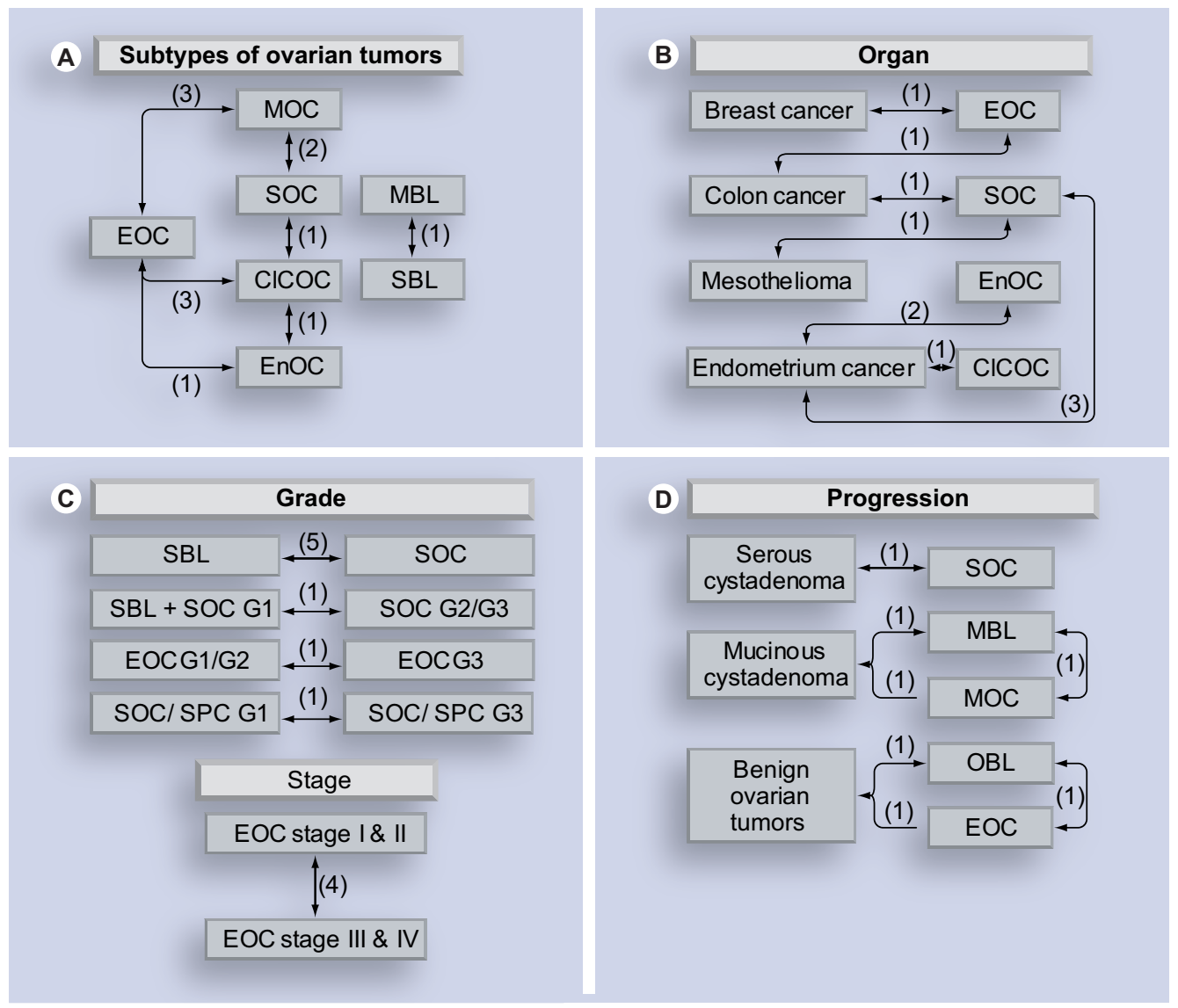

Figure 5. Genomic profiling studies in ovarian cancer demonstrating distinct entities.

Number of genomic profiling studies showing a genetic difference within groups of diseases studied (arrow with number of studies expressing a difference in brackets). Analyses are performed for (A) histological subtypes of ovarian tumors; (B) organ of disease origin; (C) grade and stage of ovarian cancer; (D) benign and malignant tumors within possible progression models of ovarian cancer.

CICOC: Clear cell ovarian cancer; EnOC: Endometrioid ovarian cancer; EOC: Epithelial ovarian cancer; MBL: Mucinous borderline tumor; MOC: Mucinous ovarian cancer; SBL: Serous borderline tumor; SOC: Serous ovarian cancer.

cancers, which have been further molecularly distinguished as early (FIGO stage I/II) or late (FIGO stage III/IV) stage disease.

\section{Aberrantly expressed genes identified across multiple transcriptomic studies}

Candidate genes differentially expressed in ovarian cancer compared with normal controls, detected by all transcriptomic studies independent of specific research question, are listed in order of their number of overlaps across studies (Table 1). Most transcriptomic profiling studies identified OI4, VEGF and $T F C P 2$, from which especially $O I 4$ and VEGF have interesting implications. Both gene products are extracellulary localized, are glycosylated and are used as targets for therapies which are either already clinically used; bevacizumab for VEGF; gefitinib for EGFR) or in clinical
Phase II/III trials, targeting VEGF, EGFR, CLDN4 and HER3. Other less frequently identified biomolecules show involvement of either cellular communication, adhesion or immune response. Post-translational modifications, especially glycosylation modifications, can be found in $55 \%$ of these biomolecules.

We have also identified overexpressed genes overlapping across studies addressing the same specific research question. Our approach was only possible for analyses of carcinoma versus normal, subtypes, grades and treatment. In this subquestion examination, a maximum of five overlaps across studies could be identified for individual genes (Table 2). A reason for this low number is the diversity of clinical questions, which makes it difficult to give question-specific markers. Interestingly, there are some genes which persist across a number of different specific aims, namely HE4 for 


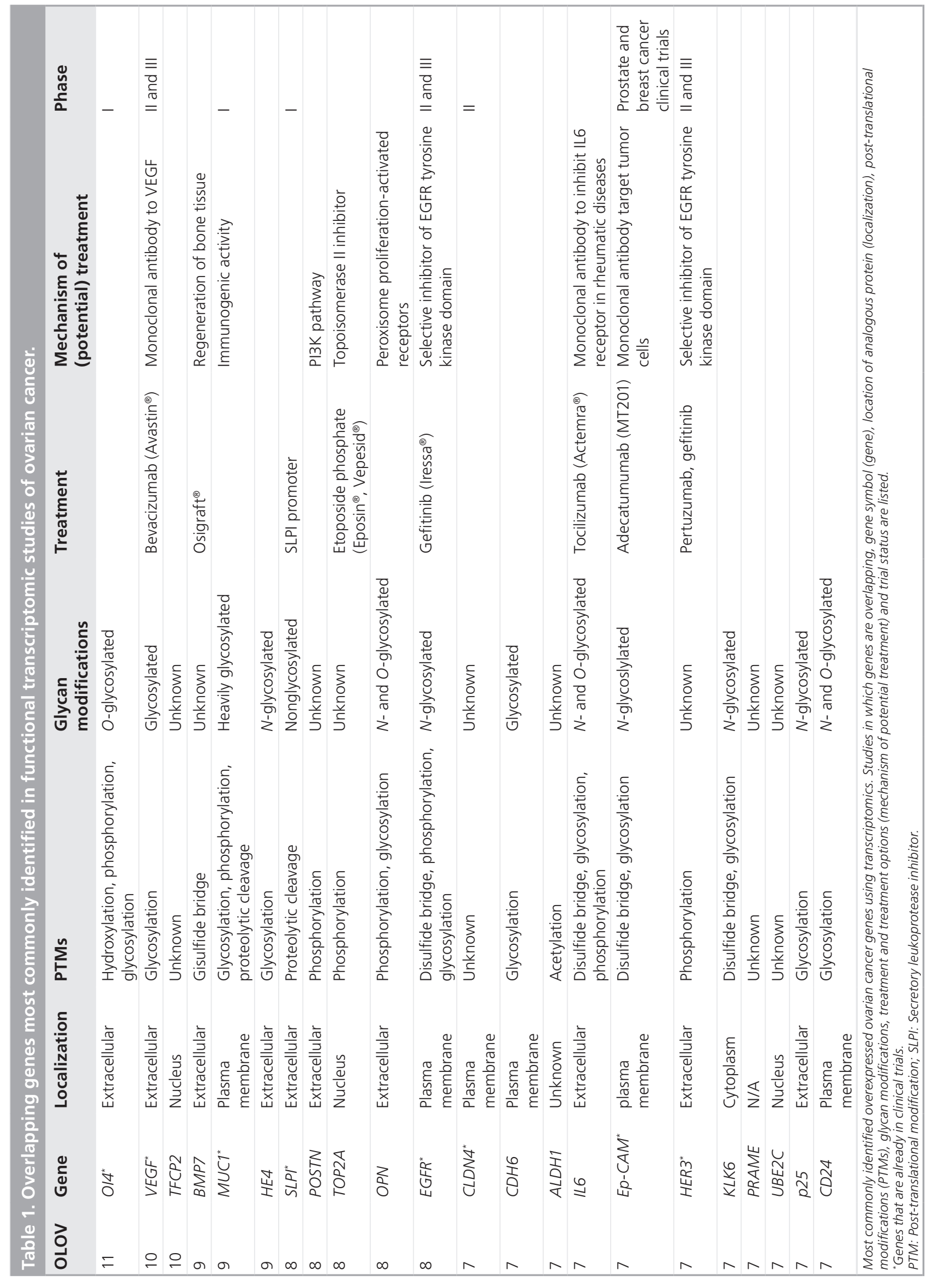


Table 2. Overlapping overexpressed genes regarding study question.

\section{Research aim}

Carcinoma vs normal (maximum overlaps 5)

Subtypes (maximum overlaps 4)

Grades (maximum overlaps 3)

Treatment (maximum overlaps 4)

\section{Top candidates and number of overlaps in studies}

POSTN (5); HE4 (5); OPN (5); EP-CAM (5); ERBB3 (5); CD24 (5); MUC1 (5); UBE2C (5); CPLA2 (5); KLK7 (5); PRAME (5)

OPN (4); POSTN (4); C7 (4); CR (4)

HE4 (3); IBP5 (3); Col6A1 (3); PAX8 (3), ALP (3)

CCN1 (4); CYR61 (4); GIG1 (4) carcinoma versus normal and grades, and OPN for 'carcinoma versus normal' and 'subtypes'. Genes identified in the 'treatment' research question analysis were unique to this question only (Table 2 ).

\section{Established knowledge due to proteogenomics}

The aim to improve upon early screening techniques and to develop more effective, targeted treatments for ovarian cancer has driven the field of high-throughput technologies since their inception 10 years ago. The success of the intensive international efforts to improve outcomes, with new targeted therapies being tested in Phase III trials (e.g., VEGF inhibitors as the most advanced therapeutics), has now become evident. Moreover, the first diagnostic marker, HE4, is commercially promoted by Fujirebbio Inc. and is increasingly being used in conjunction with CA125. Particularly notable today is the appreciation of the heterogeneity of ovarian cancer, necessitating its re-categorization into multiple distinct entities. It is thus recognized that we must treat especially mucinous and clear cell ovarian cancers differently from serous and endometrioid histological subtypes. The careful reflection on the employment of proteogenomic technologies and the critical assessment of the benefits gained from them compared with their costs has also become an unquestionable factor. It is equally important to learn from mistakes and to carefully design future studies in order to maximize the benefit from these costly technologies (TAвle 3 ).

\section{Future perspective}

New, more specific high-throughput technologies under development target the biological products of the gene-protein network, namely glycosylation, phosphorylation and metabolization. These reflect post-translational modifications of ovarian cancer and therefore carry important biological information. Most of our current clinical tumor markers are glycosylated proteins (CA125, CEA and CA19-9), $55 \%$ of all newly identified biomarkers show glycosylation (TaвLe 3). Technical simplification and standardization of these new methods will improve the uniformity and comparability of results across cohorts and is expected to massively improve our knowledge of ovarian cancer.

Table 3. Optimization for future profiling studies.

\begin{tabular}{|ll}
\hline Optimization & Study design \\
\hline 1 & $\begin{array}{l}\text { Experimental design in } \\
\text { collaboration with statistician }\end{array}$ \\
\hline 3 & $\begin{array}{l}\text { Interdisciplinary team approach } \\
\text { involves: biologist, gynecological } \\
\text { oncologist, statistician, } \\
\text { bioinformatician, pathologist }\end{array}$ \\
4 & $\begin{array}{l}\text { Prospective collection of study } \\
\text { cohort }\end{array}$ \\
\hline 5 & $\begin{array}{l}\text { Result validation within } \\
\text { independent cohort (different } \\
\text { continent) } \\
\text { Inclusion of adequate statistically } \\
\text { significant numbers }\end{array}$ \\
\hline 6 & $\begin{array}{l}\text { Definition of study question reflects } \\
\text { the selection of profiling technique }\end{array}$ \\
\hline
\end{tabular}

Sample selection

Histological review by a specialized gynecological pathologist

Avoidance of mixed pathologies (histological subtypes or grades)

Microdissection of tissue (preferably by a gynecological pathologist)

Selection of adequate controls in tissue and serum

No group combination of proven distinct entities
Biomics platform Bioinformatical analysis selection

Established/robust technique

Technique able to address the study question

Reproducible results

No inter-personal variation

Full datasets available for analysis

Analysis by a specialized bioinformatician

Translational communication throughout complete process

Distribution of full datasets after analysis

Interstudy validations and meta-analyses 
During this last decade, biomics in the field of ovarian cancer has undergone a paradigm shift away from the single-disease theory towards the acceptance of disease heterogeneity. This implies the presence of different biological mechanisms and precursor lesions for the development of ovarian cancer and the requirement for differential classifications and individualized disease subtype-specific treatments. One significant consequence is that shortcomings of previous studies, such as heterogeneity of samples and methods, application of inadequate methodologies or missing bioinformatics expertise in analysing the datasets, have been largely overcome. Another consequence is that numerous tumor markers have been proposed and are now under evaluation in combination with or without CA125, the still unbeaten tumor marker for ovarian cancer.

Advantages of previous proteogenomic studies include the wealth of data available within the scientific community, the lessons learned from mistakes, and subsequent improvements in technologies and study design and analysis techniques. A far-reaching aspect of increased data acquisition is an accompanying increase in data sharing. There are now several prominent publicly accessible databases of genomic information. These include general microarray data repositories such as the Gene Expression Omnibus (GEO) [67,68], ArrayExpress [69], and the Stanford Microarray Database (SMD) [70] and cancer-specific data repositories such as the Expression Project for Oncology (expO) [106] and ONCOMINE [71,72].

The experimental design and statistical analysis of microarray studies has evolved greatly from the early days of simplistic, single-sample comparisons [73]. In addition to the ordinary considerations of any scientific study, important aspects more specific to design of microarray studies include pairing of samples for dualchannel arrays and sample processing protocols to avoid confounding and increase precision of inferences [74]. The analysis of microarray data generally includes a technology-specific component (so-called 'low-level' analysis) and a study aim-specific ('high-level') analysis. Low-level analysis consists of data preprocessing (image analysis and normalization), required to reliably quantify fluorescence intensities for each transcript. For a review of methods for cDNA arrays, see [75]; for Affymetrix and other single-channel arrays, the most widely used methods are MAS 5 [76] and RMA [77,78]. The most commonly carried out type of higher level analysis is identification of differential expression. Many methods exist for this, but the most reliable ones involve computing a type of $t$-statistic (or $F$-statistic) based on (log) fold change and a moderated standard deviation. A widely used method is the linear modeling approach [79].

As data sharing becomes the new standard, we gain increased opportunities for knowledge synthesis. Among these is the ability to carry out statistically sound meta-analysis for clinically relevant questions. Integrated analysis of highthroughput transcriptomic studies is complex. The ONCOMINE web tool [107] has some metaanalytic capabilities; however, it provides limited flexibility. We have created a more adaptable approach for combining information across gene expression studies [80,81] but, like ONCOMINE, it still relies on having access to primary data (not just a summary p-value or ranked gene list). An extension of our methodology should allow for combination of heterogeneous data types (such as gene expression and glycomic data) rather than across just gene expression, thereby making fuller use of existing data resources.

Despite widespread acceptance and requirement of MIAME guidelines [38], data sharing is still incomplete, limiting the possibility to quantitatively combine and critically reflect on all research results.

Based on this timeline examination of recent ovarian cancer research and current technological trends, it is of major concern that only a limited number of genes are detectable in an overlap approach. This minimal number of overlaps can be explained by the limited number of available datasets, variation in study design and platforms used and diversity of research questions studied. For this field to be of more efficient assistance in identifying clinically usable biomarkers individual studies need to be comparable within each other. Based on our examination we suggest that future biomic studies meet the following requirements:

- Adequate number of samples, depending on the research question preferably from different continents (in order to avoid or address population-specific effects);

- Prospective inclusion of patients where appropriate for the study question;

- Inclusion of all presumed precursor lesions, namely ovarian surface epithelium, inclusion cysts, fimbrial end of fallopian tube epithelium;

- Inclusion of other cancers to distinguish ovarian specificity;

- Absence of mixed pathological histotypes; 
- Clear-cut study question (or hypothesis) and study groups;

- Microdissection in all tissue samples.

Of particular importance is that:

- Qualified analysts (e.g., bioinformaticians and statisticians) perform the data analyses according to internationally accepted guidelines.

Such guidelines need to be developed to establish clear requirements for data analyses. At present, MIAME compliance is widespread but not universal. The research community will need to adapt quickly to new scientific insights in ovarian cancer to avoid costly, unnecessarily repetitive studies. To this end, we encourage the establishment and support of internationally driven programs and open access platforms that guarantee the sharing of data, especially for the new technologies under current development.

\section{Financial \& competing interests disclosure}

This paper was funded by the Cancer League of the Canton of Zurich, Switzerland (to V.H.S.); Oncosuisse (0211508-2007 to V.H.S.); Swiss National Foundation (320000-12543 to V.H.S.). The authors have no other relevant affiliations or financial involvement with any organization or entity with a financial interest in or financial conflict with the subject matter or materials discussed in the manuscript apart from those disclosed.

No writing assistance was utilized in the production of this manuscript.

\section{Executive summary}

\section{Epithelial ovarian cancer is the leading cause of death from gynecological malignancies}

- Survival rate of $20 \%$ in advanced stage disease has not changed over the last 10 years, even with improvements in chemotherapy regimen.

- Increasing acceptance of the heterogeneity of ovarian cancer, which requires a disease subtype-specific treatment regimen.

- Proteogenomics will be the basis for improvements in ovarian cancer diagnosis and management in the future.

\section{High-throughput profiling technologies \& their development in ovarian cancer}

- A total of 237 proteogenomic primary studies were published on ovarian cancer during 1999-2007.

- Most studies focused on the detection of new clinical biomarkers for ovarian cancer.

- Transcriptomic (gene expression) approaches were most commonly used.

- Proteomic methods are gaining stability and reproducibility during the last few years and will have major potential in the future.

\section{Heterogeneity as the main ovarian cancer biology finding using proteogenomic high-throughput profiling}

- A distinct genomic background was found for mucinous, clear cell and endometrioid ovarian cancers as compared with serous ovarian cancers.

- Serous borderline tumors demonstrate a distinct genetic origin from high-grade, high-stage serous ovarian cancers.

- The potential ovarian cancer precursor lesion has been defined as either ovarian surface epithelium, inclusion cysts, fallopian tubal epithelium, or tubal epithelium at the fimbrial end of the fallopian tube.

\section{Overexpressed genes identified across multiple transcriptomic studies}

- Most transcriptomic profiling studies identified O/4, VEGF and TFCP2 as overexpressed genes in ovarian cancer.

- $55 \%$ of all identified transcriptomic biomarkers express glycosylation.

- Multiple identified biomarkers are already used as new targeted treatments and for diagnostic purposes.

\section{Conclusion}

- New high-profiling technologies targeting the biological products of the gene-protein network are being developed.

- Glycomics will be an area of particular interest in the development of new biomarkers in the future as most so far identified tumor markers show glycosylation changes.

- Establishment and support of internationally driven, multidisciplinary programs joining datasets and cohorts is important.

- Study design requirements are: question-driven prospectively collected clinical samples incorporating all proposed precursor lesions, clear histological subtypes generated using microdissection.

- Future considerations include the definition of bioinformatical analysis standards and integration of different data types, the development of internationally accepted guidelines for bioinformatical analyses using qualified analysts, and open access platforms requiring full data distribution.

\section{Bibliography}

Papers of special note have been highlighted as:

- of interest

" " of considerable interest

1 Ozols RF: Challenges for chemotherapy in ovarian cancer. Ann. Oncol. 17(Suppl. 5), V181-V187 (2006).

2 Fishman DA, Bozorgi K: The scientific basis of early detection of epithelial ovarian cancer: the National Ovarian Cancer Early Detection Program (NOCEDP). Cancer Treat. Res. 107, 3-28 (2002).

3 Ozols RF: Systemic therapy for ovarian cancer: current status and new treatments. Semin. Oncol. 33, S3-S11 (2006).

4 Agarwal R, Linch M, Kaye SB: Novel therapeutic agents in ovarian cancer. Eur. J. Surg. Oncol. 32, 875-886 (2006).
5 Gilks CB: Subclassification of ovarian surface epithelial tumors based on correlation of histologic and molecular pathologic data. Int. J. Gynecol. Pathol. 23, 200-205 (2004).

6 Hess V, A'Hern R, Nasiri N et al.: Mucinous epithelial ovarian cancer: a separate entity requiring specific treatment. J. Clin. Oncol. 22, 1040-1044 (2004) 
7 Heinzelmann-Schwarz VA, Gardiner-Garden M, Henshall SM et al.: A distinct molecular profile associated with mucinous epithelial ovarian cancer. $\mathrm{Br}$. J. Cancer 94, 904-913 (2006).

8 Dehari R, Kurman RJ, Logani S, Shih IeM: The development of high-grade serous carcinoma from atypical proliferative (borderline) serous tumors and low-grade micropapillary serous carcinoma: a morphologic and molecular genetic analysis. Am. J. Surg. Pathol. 31, 1007-1012 (2007).

9 Kurman RJ, Shih IeM: Pathogenesis of ovarian cancer: lessons from morphology and molecular biology and their clinical implications. Int. J. Gynecol. Pathol. 27, 151-160 (2008).

10 Auersperg N, Wong AS, Choi KC, Kang SK, Leung PC: Ovarian surface epithelium: biology, endocrinology, and pathology. Endocr. Rev. 22, 255-288 (2001).

11 Kindelberger DW, Lee Y, Miron A et al.: Intraepithelial carcinoma of the fimbria and pelvic serous carcinoma: evidence for a causal relationship. Am. J. Surg. Pathol. 31, 161-169 (2007).

12 Lee Y, Miron A, Drapkin R et al.: A candidate precursor to serous carcinoma that originates in the distal fallopian tube. J. Pathol. 211, 26-35 (2007).

"I Identification of the fimbrial end of the fallopian tube as a precursor lesion in BRCA-based serous ovarian cancers.

13 Jarboe E, Folkins A, Nucci MR et al.: Serous carcinogenesis in the fallopian tube: a descriptive classification. Int. J. Gynecol. Pathol. 27, 1-9 (2008).

14 Salvador S, Rempel A, Soslow RA, Gilks B, Huntsman D, Miller D: Chromosomal instability in fallopian tube precursor lesions of serous carcinoma and frequent monoclonality of synchronous ovarian and fallopian tube mucosal serous carcinoma. Gynecol. Oncol. 110(3), 408-417 (2008).

15 Marcelis CL, van der Putten HW, Tops C, Lutgens LC, Moog U: Chemotherapy resistant ovarian cancer in carriers of an hMSH2 mutation? Fam. Cancer 1, 107-109 (2001).

16 Weberpals JI, Clark-Knowles KV, Vanderhyden BC: Sporadic epithelial ovarian cancer: clinical relevance of BRCA1 inhibition in the DNA damage and repair pathway. J. Clin. Oncol. 26, 3259-3267 (2008).

17 Cass I, Baldwin RL, Varkey T, Moslehi R, Narod SA, Karlan BY: Improved survival in women with BRCA-associated ovarian carcinoma. Cancer 97, 2187-2195 (2003).
18 Lawrenson K, Gayther SA: Ovarian cancer: a clinical challenge that needs some basic answers. PLoS Med. 6, E25 (2009).

19 Akahira JI, Yoshikawa H, Shimizu Y et al: Prognostic factors of stage IV epithelial ovarian cancer: a multicenter retrospective study. Gynecol. Oncol. 81, 398-403 (2001).

20 Crawford SC, Vasey PA, Paul J, Hay A, Davis JA, Kaye SB: Does aggressive surgery only benefit patients with less advanced ovarian cancer? Results from an international comparison within the SCOTROC-1 Trial. J. Clin. Oncol. 23, 8802-8811 (2005).

21 Hacker NF, Berek JS, Lagasse LD, Nieberg RK, Elashoff RM: Primary cytoreductive surgery for epithelial ovarian cancer. Obstet. Gynecol. 61, 413-420 (1983).

22 Eltabbakh GH, Mount SL, Beatty B, Simmons-Arnold L, Cooper K, Morgan A: Factors associated with cytoreducibility among women with ovarian carcinoma. Gynecol. Oncol. 95, 377-383 (2004).

23 Bhoola S, Hoskins WJ: Diagnosis and management of epithelial ovarian cancer. Obstet. Gynecol. 107, 1399-1410 (2006).

24 Friedlander M, Buck M, Wyld D et al.: Phase II study of carboplatin followed by sequential gemcitabine and paclitaxel as first-line treatment for advanced ovarian cancer. Int. J. Gynecol. Cancer 17, 350-358 (2007).

25 Carr KM, Rosenblatt K, Petricoin EF, Liotta LA: Genomic and proteomic approaches for studying human cancer: prospects for true patient-tailored therapy. Hum. Genomics 1, 134-140 (2004).

26 Yap TA, Carden CP, Kaye SB: Beyond chemotherapy: targeted therapies in ovarian cancer. Nat. Rev. Cancer 9, 167-181 (2009).

27 Sotiriou C, Neo SY, McShane LM et al.: Breast cancer classification and prognosis based on gene expression profiles from a population-based study. PNAS 100, 10393-10398 (2003).

28 Bhojwani D, Kang H, Menezes RX et al.: Gene expression signatures predictive of early response and outcome in high-risk childhood acute lymphoplastic leukemia: a Children's Oncology Group Study. J. Clin. Oncol. 26, 4376-4384 (2008)

29 Schena M, Shalon D, Davis RW, Brown PO: Quantitative monitoring of gene expression patterns with a complementary DNA microarray. Science 270, 467-470 (1995).

30 Shalon D, Smith SJ, Brown PO: A DNA microarray system for analyzing complex DNA samples using two-color fluorescent probe hybridization. Genome Res. 6, 639-645 (1996).
31 Afshari CA, Nuwaysir EF, Barrett JC: Application of complementary DNA microarray technology to carcinogen identification, toxicology, and drug safety evaluation. Cancer Res. 59, 4759-4760 (1999).

32 Naora H, Montz FJ, Chai CY, Roden RB: Aberrant expression of homeobox gene HOXA7 is associated with mullerian-like differentiation of epithelial ovarian tumors and the generation of a specific autologous antibody response. Proc. Natl Acad. Sci. USA 98, 15209-15214 (2001).

33 Schummer M, Ng WV, Bumgarner RE et al.: Comparative hybridization of an array of 21,500 ovarian cDNAs for the discovery of genes overexpressed in ovarian carcinomas. Gene 238, 375-385 (1999).

34 Duan Z, Feller AJ, Penson RT, Chabner BA, Seiden MV: Discovery of differentially expressed genes associated with paclitaxel resistance using cDNA array technology: analysis of interleukin (IL) 6, IL-8, and monocyte chemotactic protein 1 in the paclitaxel-resistant phenotype. Clin. Cancer Res. 5, 3445-3453 (1999).

35 Wang K, Gan L, Jeffery E et al.: Monitoring gene expression profile changes in ovarian carcinomas using cDNA microarray. Gene 229, 101-108 (1999).

36 Pandey A, Mann M: Proteomics to study genes and genomes. Nature 405, 837-846 (2000).

37 Heinzelmann-Schwarz VA, Gardiner-Garden M, Henshall SM et al.: Overexpression of the cell adhesion molecules DDR1, Claudin 3, and Ep-CAM in metaplastic ovarian epithelium and ovarian cancer. Clin. Cancer Res. 10, 4427-4436 (2004).

38 Knudsen TB, Daston GP: MIAME guidelines. Reprod Toxicol 19, 263 (2005).

39 Brazma A, Hingamp P, Quackenbush J et al.: Minimum information about a microarray experiment (MIAME)-toward standards for microarray data. Nat. Genet. 29, 365-371 (2001).

40 Annunziata CM, Azad N, Dhamoon AS, Whiteley G, Kohn EC: Ovarian cancer in the proteomics era. Int. J. Gynecol. Cancer 18(Suppl. 1), 1-6 (2008).

41 Kohn EC, Azad N, Annunziata C, Dhamoon AS, Whiteley G: Proteomics as a tool for biomarker discovery. Dis. Markers 23, 411-417 (2007).

42 Nossov V, Amneus M, Su F et al.: The early detection of ovarian cancer: from traditional methods to proteomics. Can we really do better than serum CA-125? Am. J. Obstet. Gynecol. 199, 215-223 (2008). 
43 Sasaroli D, Coukos G, Scholler N: Beyond CA125: the coming of age of ovarian cancer biomarkers. Biomark. Med. 3, 275-288 (2009).

- Recent review describing methods and identified candidates of ovarian cancer biomarker discovery.

44 Tung CS, Wong KK, Mok SC: Biomarker discovery in ovarian cancer. Women's Health (Lond. Engl.) 4, 27-40 (2008).

45 Hanash S: Disease proteomics. Nature 422, 226-232 (2003).

46 Schuchardt S, Sickmann A: Protein identification using mass spectrometry: a method overview. EXS 97, 141-170 (2007).

47 Biron DG, Brun C, Lefevre T et al: The pitfalls of proteomics experiments without the correct use of bioinformatics tools. Proteomics 6, 5577-5596 (2006).

48 Ting L, Cowley MJ, Hoon SL, Guilhaus M, Raftery MJ, Cavicchioli R: Normalization and statistical analysis of quantitative proteomics data generated by metabolic labeling. Mol. Cell Proteomics (2009).

49 Domon B, Aebersold R: Mass spectrometry and protein analysis. Science 312, 212-217 (2006).

50 Aebersold R,M Mann: Mass spectrometrybased proteomics. Nature 422, 198-207 (2003).

51 Ross PL, Huang YN, Marchese JN et al.: Multiplexed protein quantitation in Saccharomyces cerevisiae using aminereactive isobaric tagging reagents. Mol. Cell Proteomics 3, 1154-1169 (2004).

52 Ong ES, Len SM, Lee AC: Differential protein expression of the inhibitory effects of a standardized extract from Scutellariae radix in liver cancer cell lines using liquid chromatography and tandem mass spectrometry. J. Agric. Food Chem. 53, 8-16 (2005).

53 Pierce JD, Fakhari M, Works KV, Pierce JT, Clancy RL: Understanding proteomics. Nurs. Health Sci. 9, 54-60 (2007).

54 Schmidt A, Kellermann J, Lottspeich F: A novel strategy for quantitative proteomics using isotope-coded protein labels. Proteomics 5, 4-15 (2005).

55 Cox J, Hubner NC, Mann M: How much peptide sequence information is contained in ion trap tandem mass spectra? J. Am. Soc. Mass Spectrom. 19, 1813-1820 (2008).

56 Mueller M, Peters FT, Ricaurte GA, Maurer HH: Validated liquid chromatographicelectrospray ionization mass spectrometric assay for simultaneous determination of 3,4-methylenedioxymethamphetamine and its metabolites 3,4-methylenedioxyamphetamine, 3,4-dihydroxymethamphetamine, and 4-hydroxy-3-methoxymethamphetamine in squirrel monkey plasma. J. Chromatogr. B Analyt. Technol. Biomed. Life Sci. 855, 262-270 (2007).

57 Sturm S, Schinnerl J, Greger H, Stuppner H: Nonaqueous capillary electrophoresis-electrospray ionization-ion trap-mass spectrometry analysis of pyrroloand pyrido[1,2-a] azepine alkaloids in Stemona. Electrophoresis 29, 2079-2087 (2008).

58 Wollscheid B, Bausch-Fluck D, Henderson C et al:: Mass-spectrometric identification and relative quantification of $\mathrm{N}$-linked cell surface glycoproteins. Nat. Biotechnol. 27, 378-386 (2009).

59 Keshishian H, Addona T, Burgess M, Kuhn E, Carr SA: Quantitative, multiplexed assays for low abundance proteins in plasma by targeted mass spectrometry and stable isotope dilution. Mol. Cell Proteomics 6, 2212-2229 (2007).

60 Lange V, Picotti P, Domon B, Aebersold R: Selected reaction monitoring for quantitative proteomics: a tutorial. Mol. Syst. Biol. 4, 222 (2008).

61 Whiteaker JR, Zhang H, Zhao L et al.: Integrated pipeline for mass spectrometrybased discovery and confirmation of biomarkers demonstrated in a mouse model of breast cancer. J. Proteome Res. 6, 3962-3975 (2007).

62 MacBeath G: Protein microarrays and proteomics. Nat. Genet. 32(Suppl.), 526-532 (2002).

63 Simone NL, Bonner RF, Gillespie JW, Emmert-Buck MR, Liotta LA: Laser-capture microdissection: opening the microscopic frontier to molecular analysis. Trends Genet. 14, 272-276 (1998).

64 Hergenhahn M, Kenzelmann M, Grone HJ: Laser-controlled microdissection of tissues opens a window of new opportunities. Pathol. Res. Pract. 199, 419-423 (2003).

65 Lemaire R, Menguellet SA, Stauber J et al: Specific MALDI imaging and profiling for biomarker hunting and validation: fragment of the $11 \mathrm{~S}$ proteasome activator complex, Reg $\alpha$ fragment, is a new potential ovary cancer biomarker. J. Proteome Res. 6, 4127-4134 (2007).

66 Jarboe EA, Folkins AK, Drapkin R, Ince TA, Agoston ES, Crum CP: Tubal and ovarian pathways to pelvic epithelial cancer: a pathological perspective. Histopathology 53(2), 127-138 (2008).

67 Barrett T, Suzek TO, Troup DB et al.: NCBI GEO: mining millions of expression profiles - database and tools. Nucleic Acids Res. 33, D562-D566 (2005).
68 Edgar R, Domrachev M, Lash AE: Gene expression omnibus: $N C B I$ gene expression and hybridization array data repository. Nucleic Acids Res. 30, 207-210 (2002).

69 Parkinson H, Kapushesky M, Kolesnikov N et al:: ArrayExpress update - from an archive of functional genomics experiments to the atlas of gene expression. Nucleic Acids Res. 37, D868-D872 (2009).

70 Demeter J, Beauheim C, Gollub J et al.: The Stanford Microarray Database: implementation of new analysis tools and open source release of software. Nucleic Acids Res. 35, D766-D770 (2007).

71 Rhodes DR, Kalyana-Sundaram S, Mahavisno V et al.: ONCOMINE 3.0: genes, pathways, and networks in a collection of 18,000 cancer gene expression profiles. Neoplasia 9, 166-180 (2007).

- Updates the contents and functionality of ONCOMINE.

72 Rhodes DR, Yu J, Shanker K et al: ONCOMINE: a cancer microarray database and integrated data-mining platform. Neoplasia 6, 1-6 (2004).

- Describes the contents and functionality of ONCOMINE.

73 Goldstein DR, Delorenzi M: Statistical design and data analysis for microarray experiments. In: Unraveling Lipid Metabolism with Microarrays. Berger A, Roberts MA (Eds). Dekker, New York, USA (2004).

74 Yang YH, Speed T: Design issues for cDNA microarray experiments. Nat. Rev. Genet. 3 , 579-588 (2002).

75 Smyth GK, Speed T: Normalization of cDNA microarray data. Methods 31, 265-273 (2003).

76 Affymetrix: Affymetrix Microarray Suite User Guide. Affymetrix, Santa Clara, CA, USA (2002).

77 Irizarry RA, Bolstad BM, Collin F, Cope LM, Hobbs B, Speed TP: Summaries of Affymetrix GeneChip probe level data. Nucleic Acids Res. 31, E15 (2003).

78 Irizarry RA, Hobbs B, Collin F et al.: Exploration, normalization, and summaries of high density oligonucleotide array probe level data. Biostatistics 4, 249-264 (2003).

79 Smyth GK: Linear models and empirical Bayes methods for assessing differential expression in microarray experiments. Stat. Appl. Genet. Mol. Biol. 3, 3 (2004).

80 Wirapati P, Goldstein DR, Delorenzi M: Integrated analysis of gene expression profiling studies - examples in breast cancer. In: Bioinformatics: a Swiss Perspective. Appel RD, Feytmans E (Eds). World Scientific Pub Co., Singapore (2009). 
- Details a pipeline for meta-analysis of microarray data, and illustrates the methods with two breast cancer examples.

81 Wirapati P, Sotiriou C, Kunkel S et al.: Meta-analysis of gene expression profiles in breast cancer: toward a unified understanding of breast cancer subtyping and prognosis signatures. Breast Cancer Res. 10, R65 (2008).

" Example of meta-analysis of gene expression profiles in breast cancer with both the analytic and biological perspectives.

\section{Websites}

101 US National Library of Medicine: Pubmed www.ncbi.nlm.nih.gov/pubmed

101 The Cochrane Collaboration www.cochrane.org

101 Medline $^{\circledR}$

http://medline.cos.com

101 GeneCards ${ }^{\circledR}$ Human Gene Database www.genecards.org
101 Human Protein Reference Database www.hprd.org

101 Expression Project for Oncology www.intgen.org/expo.cfm

101 ONCOMINE $^{\mathrm{TM}}$ Research www.oncomine.org 